\title{
Value distribution for difference operator of meromorphic functions with maximal deficiency sum
}

\section{Zhaojun $\mathrm{Wu}^{*}$}

${ }^{*}$ Correspondence:

wuzj52@hotmail.com

School of Mathematics and

Statistics, Hubei University of

Science and Technology, Xianning,

437100, P.R. China

\begin{abstract}
The main purpose of this paper is to investigate the relationship between the characteristic function of a meromorphic function $f(z)$ with maximal deficiency sum and that of the exact difference $\Delta_{c} f=f(z+c)-f(z)$. As an application, the author also establishes an inequality on the zeros and poles for $\Delta_{c} f$ and gives an example to show that the upper bound of the inequality is accurate.
\end{abstract}

MSC: 30D30; 39A05

Keywords: difference operator; maximal deficiency sum; meromorphic function

\section{Introduction}

If $f(z)$ is a meromorphic function in the complex plane $\mathbb{C}$ and $a \in \mathbb{C}$, we use the following notations frequently used in Nevanlinna theory (see [1-3]): $m(r, f), N(r, f), m(r, a)=$ $m\left(r, \frac{1}{f-a}\right), N(r, a)=N\left(r, \frac{1}{f-a}\right), \ldots$. Denote by $S(r, f)$ any quantity such that $S(r, f)=o(T(r, f))$, $r \rightarrow \infty$ without restriction if $f(z)$ is of finite order and otherwise except possibly for a set of values of $r$ of finite linear measure. The Nevanlinna deficiency of $f$ with respect to a finite complex number $a$ is defined by

$$
\delta(a, f)=\liminf _{r \rightarrow \infty} \frac{m(r, a)}{T(r, f)}=1-\limsup _{r \rightarrow \infty} \frac{N(r, a)}{T(r, f)} .
$$

If $a=\infty$, then one should replace $m(r, a)(N(r, a))$ in the above formula by $m(r, f)(N(r, f))$. The classical second fundamental theorem of Nevanlinna theory asserts that the total deficiency of any meromorphic function $f(z)$ satisfies the inequality

$$
\sum_{a \in \mathbb{C}} \delta(a, f)+\delta(\infty, f) \leq 2
$$

If the above equality holds, then we say that $f$ has maximal deficiency sum. The ValironMo'honko identity states that if the function $R(z, f)$ is rational in $f$ and has small meromorphic coefficients, then

$$
T(r, R(z, f))=\operatorname{deg}_{f}(R) T(r, f)+S(r, f) .
$$

Certain relationship between the characteristic function of a meromorphic function $f(z)$ with maximal deficiency sum and that of derivative $f^{\prime}(z)$ plays a key role in the study of a

\section{Springer}

o2013 Wu; licensee Springer. This is an Open Access article distributed under the terms of the Creative Commons Attribution License (http://creativecommons.org/licenses/by/2.0), which permits unrestricted use, distribution, and reproduction in any medium, provided the original work is properly cited. 
conjecture of Nevanlinna (see [4]). The main contribution of this paper is to study the relationship between the characteristic function of a meromorphic function $f(z)$ with maximal deficiency sum and that of the exact difference $\Delta_{c} f=f(z+c)-f(z)$, where $c \neq 0$ (see [5]).

In 1956, Shan and Singh [6] proved the following theorem.

Theorem A [6] Suppose that $f(z)$ is a transcendental meromorphic function of finite order and $\sum_{a \in \mathbb{C}} \delta(a, f)=2$. Then

$$
T\left(r, f^{\prime}\right) \sim 2 T(r, f), \quad r \rightarrow+\infty .
$$

After that, Edrei [7] and Weitsman [4] proved the following theorem, respectively.

Theorem B $[4,7]$ Suppose that $f(z)$ is a transcendental meromorphic function of finite order with maximal deficiency sum. Then

$$
\lim _{r \rightarrow+\infty} \frac{T\left(r, f^{\prime}\right)}{T(r, f)}=2-\delta(\infty, f)
$$

and

$$
\lim _{r \rightarrow+\infty} \frac{N\left(r, \frac{1}{f^{\prime}}\right)}{T\left(r, f^{\prime}\right)}=0 .
$$

Under the condition of Theorem B, Singh and Gopalakrishna [8] proved that

$$
\lim _{r \rightarrow+\infty} \frac{N(r, a)}{T(r, f)}=1-\delta(a, f)
$$

holds for every $a \in \mathbb{C}$.

Let $f(z)$ be a transcendental meromorphic function of order less than one. Bergweiler and Langley [9] proved that $\Delta_{c} f(z) \sim f^{\prime}(z)$ outside some exceptional set. Motivated by this result, we extend Theorem $\mathrm{B}$ to the exact difference $\Delta_{c} f$ and prove the following theorem.

Theorem 1.1 (main) Suppose that $f(z)$ is a transcendental meromorphic function of order less than one with maximal deficiency sum. Then we have

(1) $\lim _{r \rightarrow+\infty} \frac{T\left(r, \Delta_{f} f\right)}{T(r, f)}=2-\delta(\infty, f)$.

(2) $\lim _{r \rightarrow+\infty} \frac{N\left(r, \frac{1}{\Delta c f}\right)}{T\left(r, \Delta_{c} f\right)}=0$.

Consequently, we have that the deficiency of $\Delta_{c} f$ with respect to 0 is 1, i.e.,

$$
\delta\left(0, \Delta_{c} f\right)=1-\limsup _{r \rightarrow+\infty} \frac{N\left(r, \frac{1}{\Delta_{c} f}\right)}{T\left(r, \Delta_{c} f\right)}=1 .
$$

For the zeros and poles involving the derivative of a transcendental meromorphic function of finite order with maximal deficiency sum, Singh and Kulkarni [10] proved the following theorem.

Theorem C [10] Suppose that $f(z)$ is a transcendental meromorphic function of finite order with maximal deficiency sum. Then

$$
\frac{1-\delta(\infty, f)}{2-\delta(\infty, f)} \leq K\left(f^{\prime}\right) \leq \frac{2(1-\delta(\infty, f))}{2-\delta(\infty, f)},
$$


where

$$
K\left(f^{\prime}\right)=\limsup _{r \rightarrow+\infty} \frac{N\left(r, f^{\prime}\right)+N\left(r, \frac{1}{f^{\prime}}\right)}{T\left(r, f^{\prime}\right)} .
$$

In 2000, Fang [11] proved the following theorem.

Theorem D [11] Suppose that $f(z)$ is a transcendental meromorphic function of finite order with maximal deficiency sum. Then

$$
K\left(f^{\prime}\right)=\frac{2(1-\delta(\infty, f))}{2-\delta(\infty, f)} .
$$

In fact, Fang [11] proved that Theorem $D$ is valid for higher order derivatives of $f(z)$. In this paper, we shall extend Theorem $\mathrm{D}$ to the exact difference $\Delta_{c} f$ and prove the following theorem.

Theorem 1.2 (main) Suppose that $f(z)$ is a transcendental meromorphic function of order less than one with maximal deficiency sum. Then

$$
K\left(\Delta_{f} f\right) \leq \frac{2(1-\delta(\infty, f))}{2-\delta(\infty, f)}
$$

where

$$
K\left(\Delta_{c} f\right)=\limsup _{r \rightarrow+\infty} \frac{N\left(r, \Delta_{c} f\right)+N\left(r, \frac{1}{\Delta_{c} f}\right)}{T\left(r, \Delta_{c} f\right)} .
$$

The following example shows that the upper bound of the inequality in Theorem 1.2 is accurate.

Example 1.3 Let $f(z)=\frac{1}{e^{z}-1}$, then $\Delta_{c} f=\frac{\left(1-e^{c}\right) e^{z}}{\left(e^{c} e^{z}-1\right)\left(e^{z}-1\right)}$. Then $\Delta_{c} f \neq 0, \delta(0, f)=1, \delta(-1, f)=$ $1, \delta(\infty, f)=0$. Thus $f(z)$ is a meromorphic function with maximal deficiency sum. It is obvious that $\delta\left(0, e^{z}\right)=1, \delta\left(\infty, e^{z}\right)=1$ and $N\left(r, \Delta_{c} f\right)=N\left(r, e^{z}=1\right)+N\left(r, e^{z}=e^{-c}\right)$. It follows from (1.2) that

$$
N\left(r, e^{z}=1\right)=N\left(r, e^{z}=e^{-c}\right) \sim T\left(r, e^{z}\right), \quad r \rightarrow+\infty
$$

and from Valiron-Mo'honko identity (1.1) that

$$
T\left(r, \Delta_{c} f\right) \sim 2 T\left(r, e^{z}\right), \quad r \rightarrow+\infty .
$$

Therefore, $K\left(\Delta_{f} f\right)=\frac{2(1-\delta(\infty, f))}{2-\delta(\infty, f)}=1$.

By Theorem D and Example 1.3, we pose the following question. 
Question 1.4 Under the condition of Theorem 1.2, can we replace $K\left(\Delta_{f} f\right) \leq \frac{2(1-\delta(\infty, f))}{2-\delta(\infty, f)}$ by $K\left(\Delta_{c} f\right)=\frac{2(1-\delta(\infty, f))}{2-\delta(\infty, f)}$ ?

Corollary 1.5 Let $f(z)$ be a transcendental meromorphic function of order less than one with maximal deficiency sum, and assume $\delta(\infty, f)=1$. Then

$$
\lim _{r \rightarrow+\infty} \frac{N\left(r, \Delta_{c} f\right)}{T\left(r, \Delta_{c} f\right)}=0
$$

Consequently, we have that the deficiency of $\Delta_{c} f$ with respect to $\infty$ is 1, i.e.,

$$
\delta\left(\infty, \Delta_{c} f\right)=1-\limsup _{r \rightarrow+\infty} \frac{N\left(r, \Delta_{c} f\right)}{T\left(r, \Delta_{c} f\right)}=1 .
$$

As the end of this paper, we shall prove the following theorem.

Theorem 1.6 (main) Let $f(z)$ be a transcendental meromorphic function of order less than one, and assume $\delta(\infty, f)=1$. Then

$$
\sum_{a \in \mathbb{C}} \delta(a, f) \leq \delta\left(0, \Delta_{c} f\right)
$$

\section{Some lemmas}

Lemma 2.1 [12] Let $f(z)$ be a meromorphic function of finite order $\sigma$, and let $c$ be a nonzero complex number. Then, for each $\varepsilon>0$, we have

$$
m\left(r, \frac{f(z+c)}{f(z)}\right)=O\left(r^{\sigma-1+\varepsilon}\right) .
$$

Lemma 2.2 Let $f(z)$ be a transcendental meromorphic function of order $\sigma(<1)$, and let c be a non-zero complex number. Then

$$
m\left(r, \frac{f(z+c)}{f(z)}\right)=o(T(r, f))=S(r, f) .
$$

Proof Since the order of $f(z)$ is less than one, then, for any $0<\varepsilon<1-\sigma$, it follows from Lemma 2.1 that

$$
m\left(r, \frac{f(z+c)}{f(z)}\right)=O\left(r^{\sigma-1+\varepsilon}\right)=O(1)
$$

Therefore, we have

$$
m\left(r, \frac{f(z+c)}{f(z)}\right)=O(1)=o(T(r, f))=S(r, f) .
$$

Lemma 2.3 [12] Let $f(z)$ be a meromorphic function with the exponent of convergence of poles $\lambda\left(\frac{1}{f}\right)=\lambda<+\infty$, and let c be a non-zero complex number. Then, for each $\varepsilon>0$, we have

$$
N(r, f(z+c))=N(r, f)+O\left(r^{\lambda-1+\varepsilon}\right)+O(\log r) .
$$


From Lemma 2.3, using a similar method as that in the proof of Lemma 2.2, we can prove the following lemma.

Lemma 2.4 Let $f(z)$ be a transcendental meromorphic function of order less than one, and let c be a non-zero complex number. Then

$$
N(r, f(z+c))=N(r, f)+S(r, f) .
$$

\section{Proof of Theorem 1.1}

Proof By combining the first main theorem of Nevanlinna theory and Lemmas 2.2, 2.4, we have

$$
\begin{aligned}
T\left(r, \Delta_{c} f\right) & =m\left(r, \Delta_{c} f\right)+N\left(r, \Delta_{c} f\right) \\
& =m\left(r, \frac{f \Delta_{c} f}{f}\right)+N\left(r, \Delta_{c} f\right) \\
& \leq m\left(r, \frac{\Delta_{c} f}{f}\right)+m(r, f)+N(r, f)+N(r, f(z+c))+O(1) \\
& =T(r, f)+N(r, f)+S(r, f) .
\end{aligned}
$$

Hence,

$$
\limsup _{r \rightarrow \infty} \frac{T\left(r, \Delta_{c} f\right)}{T(r, f)} \leq 1+\limsup _{r \rightarrow \infty} \frac{N(r, f)}{T(r, f)}=2-\delta(\infty, f) .
$$

Let $\left\{a_{i}\right\}$ be a sequence of distinct complex numbers in $\mathbb{C}$ containing all the finite deficient values of $f(z)$. For any positive $q$, define

$$
F(z)=\sum_{i=1}^{q} \frac{1}{f-a_{i}}
$$

Since $T\left(r, f(z)-a_{i}\right)=T(r, f(z))+O(1)$ and $\Delta_{c}\left(f(z)-a_{i}\right)=\Delta_{c} f(z)$, we deduce from Lemma 2.2 that

$$
m\left(r, F(z) \Delta_{c} f(z)\right) \leq \sum_{i=1}^{q} m\left(r, \frac{\Delta_{c}\left(f(z)-a_{i}\right)}{f(z)-a_{i}}\right)+\log q=S(r, f) .
$$

This relation yields

$$
m(r, F(z))=m\left(r, F(z) \Delta_{c} f(z) \frac{1}{\Delta_{c} f(z)}\right) \leq m\left(r, \frac{1}{\Delta_{c} f}\right)+S(r, f) .
$$

By combining the first main theorem of Nevanlinna theory, (3.2) and Valiron-Mo'honko identity (1.1), we have

$$
\begin{aligned}
& q T(r, f)+N\left(r, \frac{1}{\Delta_{c} f}\right) \\
& \quad=T(r, F(z))+N\left(r, \frac{1}{\Delta_{c} f}\right)+O(1)
\end{aligned}
$$




$$
\begin{aligned}
& =m(r, F(z))+N(r, F(z))+N\left(r, \frac{1}{\Delta_{c} f}\right)+O(1) \\
& \leq m(r, F(z))+N\left(r, \frac{1}{\Delta_{c} f}\right)+\sum_{i=1}^{q} N\left(r, a_{i}\right)+O(1) \\
& \leq m\left(r, \frac{1}{\Delta_{c} f}\right)+N\left(r, \frac{1}{\Delta_{c} f}\right)+\sum_{i=1}^{q} N\left(r, a_{i}\right)+S(r, f) \\
& =T\left(r, \Delta_{c} f\right)+\sum_{i=1}^{q} N\left(r, a_{i}\right)+S(r, f) .
\end{aligned}
$$

Hence,

$$
\begin{aligned}
q & \leq \liminf _{r \rightarrow \infty} \frac{T\left(r, \Delta_{c} f\right)}{T(r, f)}+\sum_{i=1}^{q} \limsup _{r \rightarrow \infty} \frac{N\left(r, a_{i}\right)}{T(r, f)} \\
& =\liminf _{r \rightarrow \infty} \frac{T\left(r, \Delta_{c} f\right)}{T(r, f)}+\sum_{i=1}^{q}\left\{1-\delta\left(a_{i}, f\right)\right\} .
\end{aligned}
$$

Thus

$$
\liminf _{r \rightarrow \infty} \frac{T\left(r, \Delta_{c} f\right)}{T(r, f)} \geq \sum_{i=1}^{q} \delta\left(a_{i}, f\right) .
$$

Since $q$ is arbitrary, we have

$$
\liminf _{r \rightarrow \infty} \frac{T\left(r, \Delta_{c} f\right)}{T(r, f)} \geq \sum_{a \in \mathbb{C}} \delta(a, f)=2-\delta(\infty, f) .
$$

Then

$$
\lim _{r \rightarrow+\infty} \frac{T\left(r, \Delta_{c} f\right)}{T(r, f)}=2-\delta(\infty, f) .
$$

On the other hand, by combining the first main theorem of Nevanlinna theory and (3.2), we have

$$
\begin{aligned}
& \sum_{i=1}^{q} m\left(r, a_{i}\right)+N\left(r, \frac{1}{\Delta_{c} f}\right) \\
& \quad \leq m(r, F(z))+N\left(r, \frac{1}{\Delta_{c} f}\right)+O(1) \\
& \quad \leq T\left(r, \Delta_{c} f\right)+S(r, f)
\end{aligned}
$$

Thus

$$
\sum_{i=1}^{q} \frac{m\left(r, a_{i}\right)}{T\left(r, \Delta_{c} f\right)}+\frac{N\left(r, \frac{1}{\Delta_{c} f}\right)}{T\left(r, \Delta_{c} f\right)} \leq 1+\frac{S(r, f)}{T\left(r, \Delta_{c} f\right)}
$$


We derive from (3.3) that

$$
\begin{aligned}
& \sum_{i=1}^{q} \liminf _{r \rightarrow+\infty} \frac{m\left(r, a_{i}\right)}{T\left(r, \Delta_{c} f\right)}+\limsup _{r \rightarrow+\infty} \frac{N\left(r, \frac{1}{\Delta_{c} f}\right)}{T\left(r, \Delta_{c} f\right)} \\
& \quad \leq 1+\limsup _{r \rightarrow+\infty} \frac{S(r, f)}{T\left(r, \Delta_{c} f\right)} \\
& \quad \leq 1+\limsup _{r \rightarrow+\infty} \frac{S(r, f)}{T(r, f)} \frac{T(r, f)}{T\left(r, \Delta_{c} f\right)} \\
& \quad=1 .
\end{aligned}
$$

Thus

$$
\begin{aligned}
1 & \geq \limsup _{r \rightarrow+\infty} \frac{N\left(r, \frac{1}{\Delta_{c} f}\right)}{T\left(r, \Delta_{c} f\right)}+\sum_{i=1}^{q} \liminf _{r \rightarrow+\infty} \frac{m\left(r, a_{i}\right)}{T\left(r, \Delta_{c} f\right)} \\
& \geq \limsup _{r \rightarrow+\infty} \frac{N\left(r, \frac{1}{\Delta_{c} f}\right)}{T\left(r, \Delta_{c} f\right)}+\sum_{i=1}^{q} \liminf _{r \rightarrow+\infty} \frac{m\left(r, a_{i}\right)}{T(r, f)} \liminf _{r \rightarrow+\infty} \frac{T(r, f)}{T\left(r, \Delta_{c} f\right)} .
\end{aligned}
$$

It follows from (3.3) that

$$
1 \geq \limsup _{r \rightarrow+\infty} \frac{N\left(r, \frac{1}{\Delta_{c} f}\right)}{T\left(r, \Delta_{c} f\right)}+\frac{\sum_{i=1}^{q} \delta\left(a_{i}, f\right)}{2-\delta(\infty, f)}
$$

Since $q$ is arbitrary, we have

$$
1 \geq \limsup _{r \rightarrow+\infty} \frac{N\left(r, \frac{1}{\Delta_{c} f}\right)}{T\left(r, \Delta_{c} f\right)}+1 .
$$

Then

$$
\limsup _{r \rightarrow+\infty} \frac{N\left(r, \frac{1}{\Delta_{c} f}\right)}{T\left(r, \Delta_{c} f\right)}=0
$$

Therefore,

$$
\lim _{r \rightarrow+\infty} \frac{N\left(r, \frac{1}{\Delta_{c} f}\right)}{T\left(r, \Delta_{c} f\right)}=0 .
$$

\section{Proof of Theorem 1.2}

Proof It follows from Lemma 2.4 that

$$
N\left(r, \Delta_{c} f\right) \leq 2 N(r, f)+S(r, f) .
$$

The above inequality implies that

$$
\frac{N\left(r, \Delta_{c} f\right)}{T\left(r, \Delta_{c} f\right)} \frac{T\left(r, \Delta_{c} f\right)}{T(r, f)} \leq 2 \frac{N(r, f)}{T(r, f)}+\frac{S(r, f)}{T(r, f)} .
$$


By Theorem 1.1(1), we have

$$
(2-\delta(\infty, f)) \limsup _{r \rightarrow+\infty} \frac{N\left(r, \Delta_{c} f\right)}{T\left(r, \Delta_{c} f\right)} \leq 2(1-\delta(\infty, f)) .
$$

Therefore,

$$
\limsup _{r \rightarrow+\infty} \frac{N\left(r, \Delta_{c} f\right)}{T\left(r, \Delta_{c} f\right)} \leq \frac{2(1-\delta(\infty, f))}{2-\delta(\infty, f)}
$$

This relation and Theorem 1.1(2) together yield

$$
K\left(\Delta_{c} f\right) \leq \frac{2(1-\delta(\infty, f))}{2-\delta(\infty, f)}
$$

\section{Proof of Theorem 1.6}

Proof If $\sum_{a \in \mathbb{C}} \delta(a, f)=0$, Theorem 1.6 is valid in this case. In the following, we assume that $\sum_{a \in \mathbb{C}} \delta(a, f)>0$. Let $\left\{a_{\mu}\right\}$ be a sequence of distinct complex numbers in $\mathbb{C}$ containing all the finite deficient values of $f(z)$. For any positive integer $q$, as we did in the proof of Theorem 1.1(2), we can get that

$$
\sum_{\mu=1}^{q} m\left(r, a_{\mu}\right)+N\left(r, \frac{1}{\Delta_{c} f}\right) \leq T\left(r, \Delta_{c} f\right)+S(r, f)
$$

holds for any $q$ finite complex numbers in $\left\{a_{\mu}\right\}$. Therefore, we have

$$
\frac{N\left(r, \frac{1}{\Delta_{c} f}\right)}{T\left(r, \Delta_{c} f\right)}+\frac{T(r, f)}{T\left(r, \Delta_{c} f\right)}\left(\frac{\sum_{\mu=1}^{q} m\left(r, a_{\mu}\right)}{T(r, f)}-o(1)\right) \leq 1, \quad r \rightarrow+\infty
$$

Hence, from (3.1) we can get

$$
\begin{aligned}
1 & \geq \limsup _{r \rightarrow+\infty}\left[\frac{N\left(r, \frac{1}{\Delta_{c} f}\right)}{T\left(r, \Delta_{f} f\right)}+\frac{T(r, f)}{T\left(r, \Delta_{c} f\right)}\left(\frac{\sum_{\mu=1}^{q} m\left(r, a_{\mu}\right)}{T(r, f)}-o(1)\right)\right] \\
& \geq \limsup _{r \rightarrow+\infty} \frac{N\left(r, \frac{1}{\Delta_{c} f}\right)}{T\left(r, \Delta_{c} f\right)}+\liminf _{r \rightarrow+\infty} \frac{T(r, f)}{T\left(r, \Delta_{c} f\right)}\left(\frac{\sum_{\mu=1}^{q} m\left(r, a_{\mu}\right)}{T(r, f)}-o(1)\right) \\
& \geq \limsup _{r \rightarrow+\infty} \frac{N\left(r, \frac{1}{\Delta_{c} f}\right)}{T\left(r, \Delta_{c} f\right)}+\liminf _{r \rightarrow+\infty} \frac{T(r, f)}{T\left(r, \Delta_{c} f\right)} \liminf _{r \rightarrow+\infty} \frac{\sum_{\mu=1}^{q} m\left(r, a_{\mu}\right)}{T(r, f)} \\
& \geq \limsup _{r \rightarrow+\infty} \frac{N\left(r, \frac{1}{\Delta_{c} f}\right)}{T\left(r, \Delta_{c} f\right)}+\frac{\sum_{j=1}^{q} \delta\left(a_{\mu}, f\right)}{2-\delta(\infty, f)} .
\end{aligned}
$$

Since $q$ is arbitrary and $\delta(\infty, f)=1$, we have

$$
\sum_{a \in \mathbb{C}} \delta(a) \leq \delta\left(0, \Delta_{c} f\right)
$$




\section{Acknowledgements}

This research was partly supported by the National Natural Science Foundation of China (Grant No. 11201395) and by the Science Foundation of Educational Commission of Hubei Province (Grant No. Q20132801, D20132804).

Received: 13 August 2013 Accepted: 30 August 2013 Published: 11 Nov 2013

\section{References}

1. Hayman, WK: Meromorphic Functions. Oxford Mathematical Monographs. Clarendon Press, Oxford (1964)

2. Yang, L: Value Distribution Theory. Translated and revised from the 1982 Chinese original. Springer, Berlin (1993)

3. Zheng, JH: Value Distribution of Meromorphic Functions. Tsinghua University Press, Beijing (2010)

4. Weitsman, A: Meromorphic functions with maximal deficiency sum and a conjecture of F. Nevanlinna. Acta Math. 123, 115-139 (1969)

5. Halburd, RG, Korhonen, RJ: Nevanlinna theory for the difference operator. Ann. Acad. Sci. Fenn., Math. 31, 463-478 (2006)

6. Shan, SM, Singh, SK: On the derivative of a meromorphic function with maximum defect. Math. Z. 65, 171-174 (1956)

7. Edrei, A: Sums of deficiencies of meromorphic functions II. J. Anal. Math. 19, 53-74 (1967)

8. Singh, SK, Gopalakrishna, HS: Exceptional values of entire and meromorphic functions. Math. Ann. 191, 121-142 (1971)

9. Bergweiler, W, Langley, JK: Zeros of differences of meromorphic functions. Math. Proc. Camb. Philos. Soc. 142, 133-147 (2007)

10. Singh, SK, Kulkarni, VN: Characteristic function of a meromorphic function and its derivative. Ann. Pol. Math. 28, 123-133 (1973)

11. Fang, ML: A note on a result of Singh and Kulkarni. Int. J. Math. Math. Sci. 23, 285-288 (2000)

12. Chiang, YM, Feng, SJ: On the Nevanlinna characteristic of $f(z+\eta)$ and difference equations in the complex plane. Ramanujan J. 16, 105-129 (2008)

13. Chen, ZX, Shon, $\mathrm{KH}$ : Value distribution of meromorphic solutions of certain difference Painleve equations. J. Math. Anal. Appl. 364, 556-566 (2010)

14. Xu, HY, Cao, TB, Liu, BX: The growth of solutions of systems of complex q-shift difference equations. Adv. Differ. Equ. 2012(216), 1-22 (2012)

10.1186/1029-242X-2013-530

Cite this article as: Wu: Value distribution for difference operator of meromorphic functions with maximal

deficiency sum. Journal of Inequalities and Applications 2013, 2013:530

\section{Submit your manuscript to a SpringerOpen ${ }^{\ominus}$ journal and benefit from:}

- Convenient online submission

- Rigorous peer review

- Immediate publication on acceptance

- Open access: articles freely available online

- High visibility within the field

- Retaining the copyright to your article 